\title{
The Use of 3D Printing in the Development of Gaseous Radiation Detectors
}

\author{
Sam Fargher, Chris Steer, and Lee Thompson
}

\begin{abstract}
Fused Deposition Modelling has been used to produce a small, single wire, Iarocci-style drift tube to demonstrate the feasibility of using the Additive Manufacturing technique to produce cheap detectors, quickly. Recent technological developments have extended the scope of Additive Manufacturing, or 3D printing, to the possibility of fabricating Gaseous Radiation Detectors, such as Single Wire Proportional Counters and Time Projection Chambers. 3D printing could allow for the production of customisable, modular detectors; that can be easily created and replaced and the possibility of printing detectors on-site in remote locations and even for outreach within schools.

The 3D printed drift tube was printed using Polylactic acid to produce a gas volume in the shape of an inverted triangular prism; base length of $28 \mathrm{~mm}$, height $24.25 \mathrm{~mm}$ and tube length $145 \mathrm{~mm}$. A stainless steel anode wire was placed in the centre of the tube, mid-print. P5 gas (95\% Argon, 5\% Methane) was used as the drift gas and a circuit was built to capacitively decouple signals from the high voltage. The signal rate and average pulse height of cosmic ray muons were measured over a range of bias voltages to characterise and prove correct operation of the printed detector.
\end{abstract}

Index Terms-3D Printing, Additive Manufacturing, Gaseous radiation Detectors, Drift Tubes.

\section{INTRODUCTION}

A DDITIVE Manufacturing (AM), or 3D printing, is a technique used to fabricate $3 \mathrm{D}$ models from $\mathrm{CAD}$ files by fusing together successive layers of material. AM was developed in the 1980's as a method of producing basic prototypes, however the available processes were expensive and slow. Recent years have seen new technologies and developments occur that have extended the scope of AM. Improved speed and accuracy of AM processes have enabled fast production of high quality, functional products; and an increase in the range of printable materials, such as flexible and electrically conductive thermoplastics, has allowed more complex designs and geometries to be fabricated. Commercialisation has also allowed Rapid Prototyping (the iterative process of trial and error to produce a working design) to become a cost-effective, accessible and convenient production method.

\section{A. Motivations}

In 2013 M. Hohlmann [1] posed a "Grand Challenge" to the HEP instrumentation community to use AM to produce particle detectors. In this white paper, Hohlmann shows the current AM capabilities still do not meet the requirements to 3D print a

Sam Fargher and Lee Thompson were with the Department of Physics and Astronomy, University of Sheffield, UK.

Chris Steer was with AWE plc, Aldermaston, UK.

Manuscript received July 15, 2017 fully-functional MicroPattern Gas Chambers, including a Gas Electron Multiplier, that have the spatial resolution needed for applications in HEP experiments. However, current AM techniques do have the necessary performance to allow Rapid Prototyping to be used in the R\& D phase of building such Gaseous Radiation Detectors (GRDs) and in the production of GRDs of a simpler design, such as Single Wire Proportional Counters (SWPCs) and Time Projection Chambers (TPCs).

\begin{tabular}{|l|c|c|}
\hline & Current capability & Performance goal \\
\hline Printing resolution in $\mathbf{x}-\mathbf{y}$ & $\sim 75 \mu \mathrm{m}$ & $\sim 1 \mu \mathrm{m}$ \\
\hline Layer thickness in $\mathbf{z}$ & $\sim 50 \mu \mathrm{m}$ & $\sim 1 \mu \mathrm{m}$ \\
\hline Print speed & $10 \mathrm{~cm} / \mathrm{s}$ & $>100 \mathrm{~cm} / \mathrm{s}$ \\
\hline Materials & $\begin{array}{c}\text { Either polymers or } \\
\text { metals }\end{array}$ & Polymer-metal composites \\
& $50 \mathrm{~cm} \times 50 \mathrm{~cm} \times 25 \mathrm{~cm}$ & $200 \mathrm{~cm} \times 100 \mathrm{~cm} \times 10 \mathrm{~cm}$ \\
\hline Object size & \multicolumn{2}{|l}{} \\
\hline
\end{tabular}

Fig. 1. A table comparing commercial AM capabilities to the performance needed to 3D print complete gaseous radiation detectors such as MicroPattern Gas Chambers (containing GEMs), for high spatial resolution detectors required in HEP experiments [1].

\section{3D PRINTING WiRED GASEOUS RADIATION DETECTORS}

Fused Deposition Modelling (FDM) is the main AM technique available to to the authors. This process works by extruding thin lines of molten thermoplastic, in the x-y plane, onto a base plate to produce a "slice" of a model. The baseplate then moves downwards in the z-axis and another "slice" is printed onto the previous, thermally fusing as the plastic is printed. This process is repeated until a 3D model is produced.

An Iarocci-style drift tube was chosen to be the first type of GRD to be printed as this is of the simplest design. Iarocci tubes are often cylindrical, a few $\mathrm{cm}$ in diameter, with an anode wire of diameter $\sim 100 \mu \mathrm{m}$, and a resistive cathode (typically graphite) coating on the inside. This internal resistive cathode allows for an external pick-up electrode to be used, allowing for easier detector construction [2]. The initial challenges of 3D printing a the drift tube using FDM are producing a hollow enclosure, as the drift tube 'roof' would have to be printed over empty space; introducing a taut anode wire in the correct position, which will remain taut; and ensuring the enclosure is airtight.

Acrylonitrile butadiene styrene (ABS) and Polylactic acid (PLA) were investigated as possible thermoplastics to print the enclosure, with a variety of different drift tube enclosure designs. Two printers were used; the Makerbot Replicator 2X for the ABS and the re:3D Gigabot (Fig. 2) for the PLA. Each 


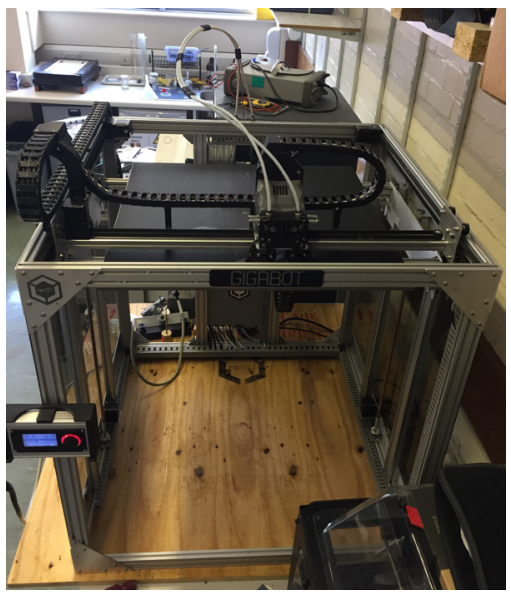

Fig. 2. Picture of the re:3D Gigabot at The University of Sheffield. The Gigabot is a large scale commercial FDM printer, with a possible print volume of dimensions $590 \times 600 \times 600 \mathrm{~mm}^{3}$.

drift tube was designed using OpenSCAD to produce an .stl file which was then converted, via slicing software, into printable .x3g or .GCODE files, depending on the printer used. The .x3g file format is a binary file used by the Makerbot and the .GCODE file format is a Computer Numerical Control (CNC) file used by the Gigabot. The .GCODE files have a significant advantage over the .x3g as GCODE is a list of commands followed by the printer; for $3 \mathrm{D}$ printing each successive layer of the print has its own section of code, allowing the possibility of editing the .GCODE before printing, to customise certain layers. From the tests, ABS was found to warp too easily and provide poor overhang formation for the majority of the enclosure designs, however PLA produced stable prints and excellent overhang formation for all tested designs and an inverse triangular prism drift tube was selected to be continued with. These results, along with the advantages that come with using .GCODE, meant using PLA with the Gigabot henceforth.

Having found PLA to be the best thermoplastic to use, a new drift tube was created using OpenSCAD. This drift tube had a base length of $28 \mathrm{~mm}$, height $24.25 \mathrm{~mm}$ and tube length $145 \mathrm{~mm}$ and included gas inlet valves (see Fig. 3 for crosssection).

A frame was also designed, and 3D printed with the Gigabot, to hold an anode wire at high tension. The height of the frame was designed to be half the height of the drift tube, allowing the wire to run through the centre of the drift tube. A stainless steel wire, $(r=50 \mu m)$, was secured to the frame using Araldite while using a $300 \mathrm{~g}$ load to supply the correct tension. Although stainless steel is not an ideal metal for a drift tube anode wire, it was the easiest available wire for these tests.

Four pegs, each $(10 \times 10 \times 5) \mathrm{mm}^{3}$, were added to the drift tube model on OpenSCAD, which corresponded to the inner corners of the wire frame (see Fig. 4). These pegs ensure the wire frame can only be placed in the correct position to leave the anode wire running central down the tube.

The drift tube .GCODE was generated, usingSimplify3D slicing software, and subsequently edited to pause the process

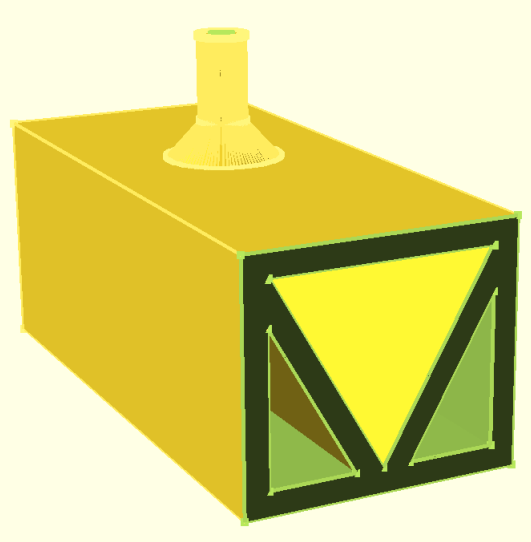

Fig. 3. Cross-section of the OpenSCAD drift tube design with gas inlet. Open sections at bottom left and right are connected to the main, central triangular prism to allow gas to flow within but these sections do not contribute to any signals.

mid-print, at the height of the wire frame; lower the baseplate; and wait for 120 seconds, allowing enough time for the frame holding the anode wire to be introduced. Once in place, the $3 \mathrm{D}$ printing process continued, depositing molten plastic over the anode wire, securing it in place. The drift tube walls were printed with a $20 \%$ infill, however this amount of plastic was not sufficient to grip the wire and maintain the tension required for operation once the wire was cut free from the frame. Instead of increasing the infill of the entire drift tube, the GCODE corresponding to the 5 printed layers, above and below the anode wire, were replaced with the .GCODE generated with $100 \%$ infill, which gripped the anode wire sufficiently.

Once printed, the anode wire was cut free from the frame, leaving a hollow drift tube with a central anode wire. Each end of the anode wire was soldered to an SHV connector (with one end connected via a $1 \mathrm{M} \Omega$ resistor) fixed to $3 \mathrm{D}$ printed end caps to provide a barrier to the bare wire, which would be connected to a high voltage (see Fig. 5). The drift tube was then coated with copper shielding spray to provide RF protection and act as an external cathode (grounded) as an internal cathode has not yet been introduced into the drift tube design.

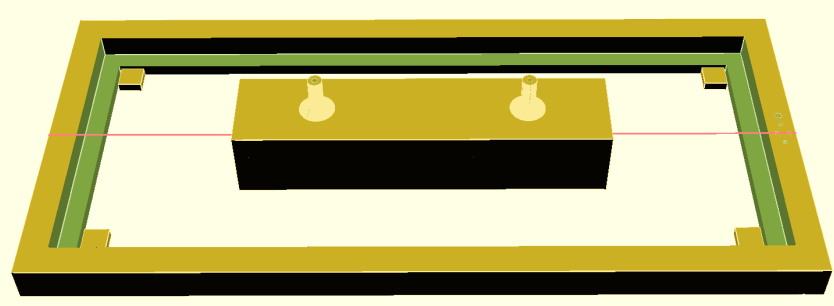

Fig. 4. OpenSCAD design of drift tube, wire rig and pegs, to allow the wire (red) to be correctly placed within the drift tube. Although all designs are collected here, the drift tube and pegs are one design and the wire rig is another design to be printed separately. The wire (red) is not part of either model but has been added to show position. 
A simple circuit was built to take the output of the drift tube and capacitively decouple any signals from the bias voltage before being passed to an oscilloscope. An airtight metal box was modified to take the gas output of the drift tube and monitor the oxygen level, using a SGX oxygen sensor, as a measure of contaminants in the drift gas. The oxygen monitor box, circuit enclosure and circuit diagram can be seen in Fig. 5 .
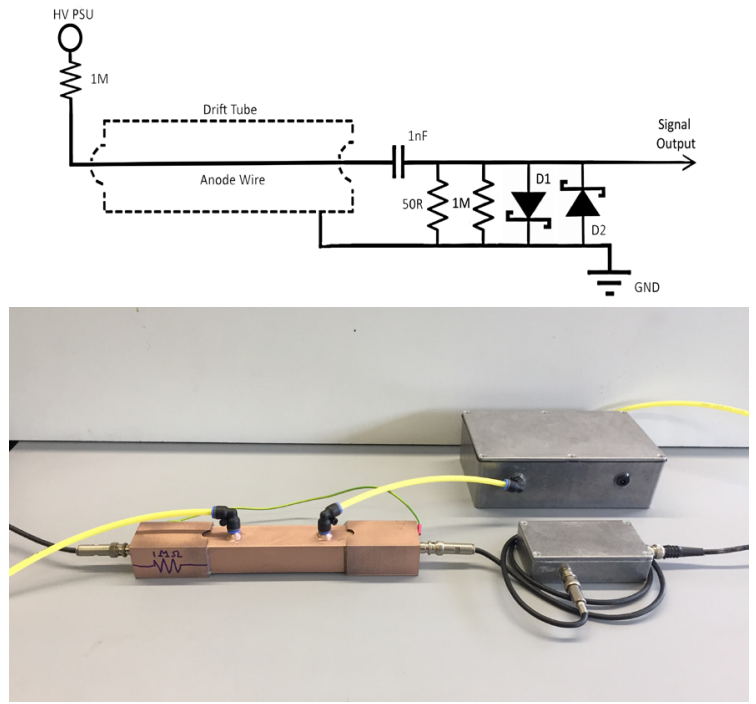

Fig. 5. UPPER: Circuit diagram of signal decoupling circuit. The $50 \Omega$ resistor was included to better match the impedance of the signal output system. Components D1 and D2 are Schottky Diodes included as a precaution, to limit the signal amplitude and protect the oscilloscope electronics. LOWER: Picture of completed drift tube connected to signal decoupling circuit (small metal box) and oxygen sensor (large metal box).

\section{Characterisation}

To characterise the drift tube, the average pulse height and signal rate was determined for a range of applied bias voltages. P5 gas (95\% Argon 5\% Methane) was flowed through the drift tube at a constant flow rate of $0.2 \mathrm{litres} / \mathrm{min}$ and the oxygen level monitored and maintained below $0.0025 \%$. The temperature of the gas was maintained at $299.0 \pm 1.5 \mathrm{~K}$. A range of bias voltages were applied to the anode wire via a V6533P 6 Channel VME Programmable HV Power Supply and the raw signals, decoupled from the bias voltage, were passed onto a Teledyne LeCroy WaveRunner Oscilloscope to record the signal pulse heights and signal rate. Cosmic ray muons were used to produce signals in the drift tube and a minimum of a few thousand signals were recorded for each of the applied bias voltages.

Fig. 6 shows the variation of signal rate against the bias voltage applied to the anode wire. These results were obtained from a relatively low statistics data run, however, the graph does show that the signal rate generally increases with bias voltage; as expected with GRDs. It should be noted that although the drift tube has been operated at high voltages, uncommon for most GRDs, these voltages are typical for Iarocci tube operation.

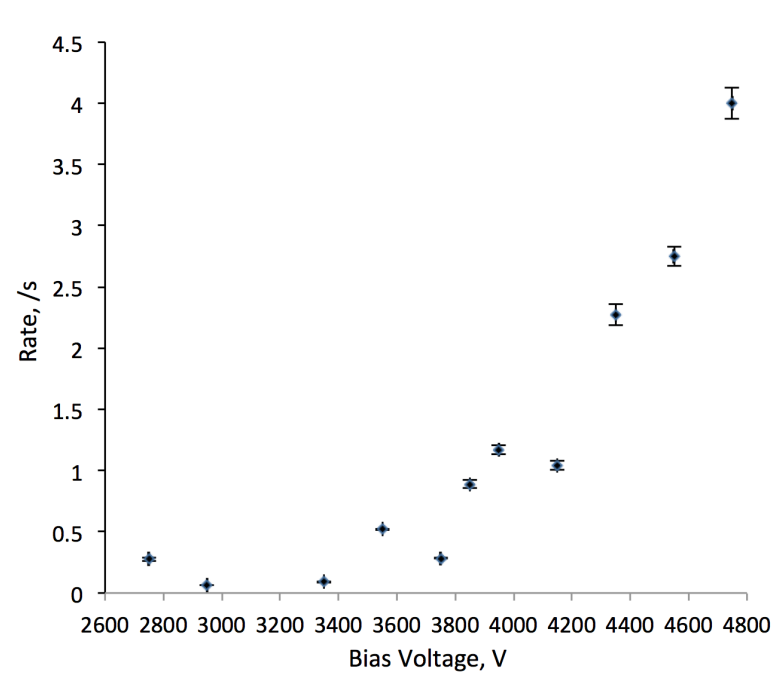

Fig. 6. Graph of average signal rate against applied bias voltage and errors.

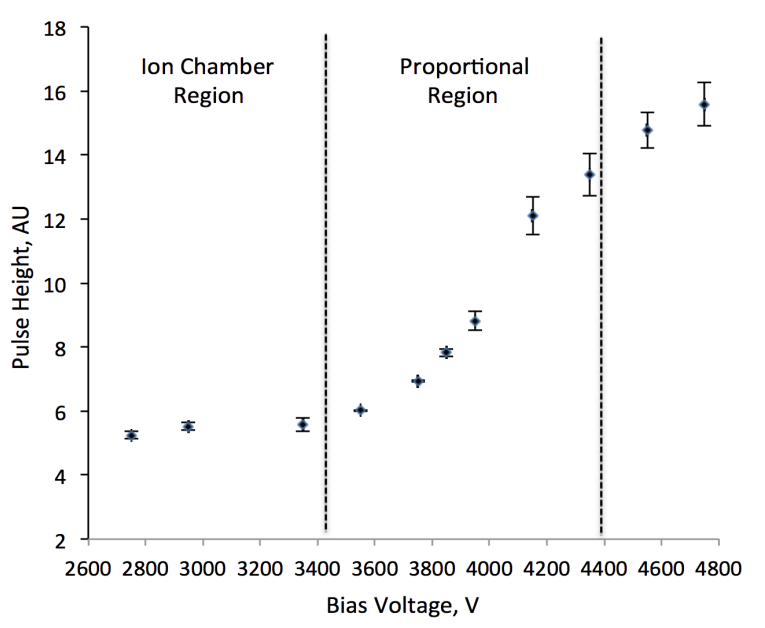

Fig. 7. Graph of pulse heights against applied bias voltage and with errors The assumed regions of operation are labelled. The pulse heights are in arbitrary units as the raw signals are used, and a integrator and shaper need to be used to fix appropriate units to values.

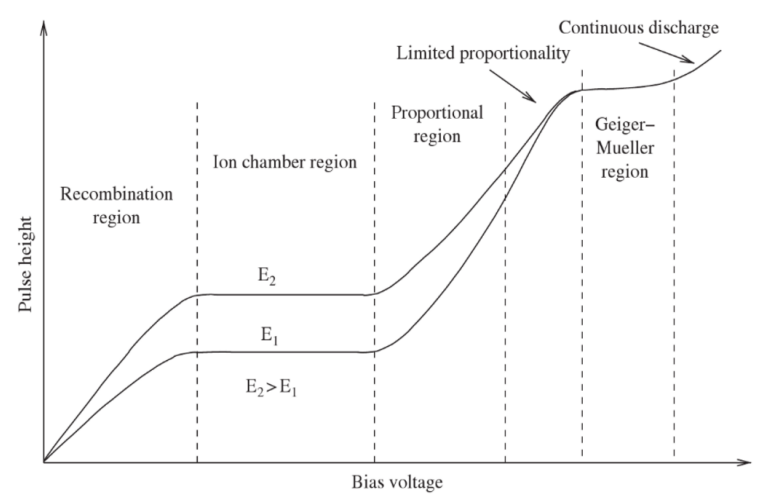

Fig. 8. Graph depicting the typical variation in average pulse height, with bias voltage, found for GRDs. The operation regions are also labelled [3]. 
Fig. 7 shows the variation in average pulse height against the bias voltage applied to the $3 \mathrm{D}$ printed drift tube anode wire, and the typical variation of average pulse height, with bias voltage, expected for GRDs are shown in Fig. 8. Currently, the range of bias voltages that the drift tube has been operated with is not sufficient to provide a clear correlation between the data and the expected variation, however, the general shape of the measured curve demonstrates the expected behaviour at lower voltages and the assumed regions of operation have been labelled on the graph, i.e. Ion Chamber Region and Proportional Region. As noted in the figure caption, only the raw signals have been used and the next stage is to add a pulse integrator and shaper to provide better signals for analysis.

\section{CONCLuSion}

A simple Iarocci-style drift tube was 3D printed, proving the concept that simple GRDs can be 3D printed at current AM capabilities. The characterisation of the drift tube is underway and the results appear to show the drift tube is operating correctly; giving reason to continue investigating $\mathrm{AM}$ as a means of producing GRDs. Upon completion of characterising the current 3D printed drift tube, the next stage of this project is to produce a method of introducing an internal cathode into the drift tube, to allow readout. If this is successful, it is hoped that the project will move forward to developing a method of printing more complex GRDs, such as Time Projection Chambers.

\section{REFERENCES}

[1] M. Hohlmann, "Printing out Particle Detectors with 3D-Printers ? a Potentially Transformational Advance for HEP Instrumentation", in APSDPF Snowmass Summer Study, The Division of Particles and Fields of the American Physical Society, 2013.

[2] E. Iarocci, "Plastic streamer tubes and their applications in high energy physics", Nuclear Instruments and Methods in Physics Research, vol. 217, no. $1-2$, pp. 30-42, 1983.

[3] S. Ahmed, "Physics And Engineering Of Radiation Detection", [S.1.]: Elsevier, 2007. 